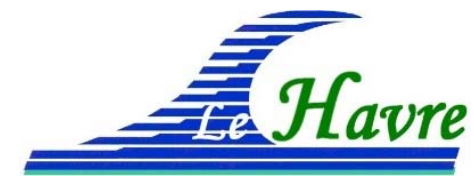

XVI èmes Journées Nationales Génie Côtier - Génie Civil

Le Havre, 2020

DOI:10.5150/jngcgc.2020.076 C Editions Paralia CFL

disponible en ligne - http://www.paralia.fr - available online

\title{
Valorisation de sédiments à teneur en eau élevée en génie côtier : construction de la digue de Xiapu (Chine)
}

\author{
Xiao WANG ${ }^{1,2}$, Daniel LEVACHER ${ }^{1}$, Tianxue ZHONG ${ }^{2}$ \\ 1. Normandie Université, Unicaen, UMR 6143 CNRS - M2C, 24 rue des Tilleuls, 14000 \\ Caen, France. \\ wangxiao@hotmail.fr ; daniel.levacher@unicaen.fr \\ 2. Jiangsu Kun Ze Technologie, Floor 5, Building B1, Ruan Jian Da Dao street, quartier \\ Du Yu Hua Tai district, Nanjing, Chine.
}

\section{Résumé :}

La rareté des matériaux naturels, la notion d'économie circulaire, la renouvelabilité des ressources en agrégats amènent les projeteurs à reconsidérer certains concepts d'ouvrages côtiers. En construction côtière, une ressource de proximité concerne les sédiments aux médiocres propriétés géomécaniques. Ces sédiments doivent être traités, stabilisés et/ou solidifiés pour acquérir des performances suffisantes pour être substitués aux matériaux naturels. A cela la nécessité de développer des nouvelles technologies de stabilisation/solidification s'avère urgente pour diminuer le temps d'exécution des projets de construction littorale, devant faire face à la rareté des matériaux naturels disponibles sur le territoire. Dans cette communication, est relatée la construction d'une partie de digue sur $200 \mathrm{~m}$ pour un projet de digue d'une longueur totale de $2 \mathrm{~km}$ sur une île. Des sédiments aux faibles propriétés sont disponibles à proximité de l'ouvrage, ils doivent être traités pour être utilisés en lieu et place de matériaux classiques prévus dans le corps de la digue. Le concept même de la digue est revu. Les sédiments sont mélangés à l'aide d'un liant, puis à l'état frais sont pompés et transportés pour remplir des sacs de géotextiles ou géotubes ${ }^{\circledR}$ et finalement se solidifient pour acquérir à 28 jours les caractéristiques exigées par le projet. Des essais de consistance sur le mélange sédimentliant à l'état frais ont été nécessaires pour assurer le transport et le remplissage des géotubes $^{\circledR}$ en relation avec le temps de prise et les conditions de chantier (température, teneur en eau du sédiment, dosage en liant). De ces essais, l'optimisation du dosage en liant a été menée pour acquérir les caractéristiques mécaniques souhaitées pour le projet de digue. Elles concernent la stabilité de l'ouvrage et sa pérennité : résistance en compression, cohésion et angle de frottement, perméabilité. Les résultats de ces essais de laboratoire ont été confrontés aux essais de contrôle effectués sur site ce qui a permis de valider la méthodologie mise en place en laboratoire et en chantier.

Mots-clés :

Sédiment, Valorisation, Liant, Propriétés mécaniques, Pompabilité, Noyau de digue, Développement durable. 


\section{Thème 6 - Gestion durable des zones littorales et estuariennes}

\section{Introduction}

Depuis une décennie, la Chine a fortement investi dans le développement et la mise en valeur des zones côtières à des fins industrielles stratégiques. Ceci s'est traduit non seulement par des aménagements côtiers mais aussi par des extensions en mer et indirectement par la mise en place de digues de protection et d'enclôture puis de remblais afin de gagner des terrains sur la mer. Ces digues quel qu'en soit le concept, sont de grandes dimensions de longueur kilométrique, elles nécessitent pour leur construction de grands volumes de matériaux. Devant le très grand nombre actuel de projets de construction de digues en Chine et une politique de protection de l'environnement plus stricte, la disponibilité de matériaux traditionnels pose problème. Pour un projet côtier comme une digue, il devient opportun pour pallier cette raréfaction de matériaux de développer des matériaux nouveaux. La ressource renouvelable à proximité des ouvrages côtiers est naturellement relative aux sédiments. Un sédiment stabilisé ou solidifié aux propriétés mécaniques maîtrisées peut se substituer aux matériaux de remplissage pour constituer un corps ou noyau de digue. Ce sédiment doit être fluide après un mélange avec des liants pour être pompé et adapté au remplissage de sacs en géotextile pouvant constituer le noyau d'une digue. Cette phase de préparation et de construction doit être entièrement automatisée. Au-delà de cette phase, le sédiment se solidifie et doit présenter des caractéristiques mécaniques suffisantes pour assurer la stabilité du noyau (PU et al., 2019) et garantir une durée de vie suffisante de la digue. La mise au point d'un sédiment solidifié pour constituer un corps de digue en sacs de géotextile a été proposée dans le cas d'un projet de construction d'une partie de la digue sur 200m sur l'île de Zhujiang (Chine), dénommé digue de Xiapu (WANG, 2019). Tout d'abord, une étude de la fluidité du mélange sédiment-liant a été menée pour cerner les paramètres de consistance et de prise à l'aide d'essais d'affaissement et de la mesure du temps de prise en fonction du dosage en liant, de la teneur en eau, de la température. S'en est suivie une deuxième étude sur le sédiment solidifié pour déterminer le dosage en liant optimal pour satisfaire les caractéristiques mécaniques à 28 jours, à savoir une résistance à la compression non confinée, une cohésion, un angle de frottement et une perméabilité répondant aux exigences demandées au niveau du projet de la digue de Xiapu. Des essais de contrôle ont eu lieu pendant la construction (état frais du mélange) et après la construction de la digue (état durci), ceux-ci ont permis de valider à la fois la technologie développée pour la préparation et la mise en place des mélanges de sédiments sur le chantier et la méthodologie menée en laboratoire à propos des essais retenus. Le projet de la digue de Xiapu est d'abord présenté au niveau du concept de la digue et les conditions environnantes, puis les résultats des essais entrepris sur les mélanges sédiment-liant à l'état frais et à l'état solidifié sont discutés en fonction des valeurs exigées au niveau du projet. Un organigramme du processus de construction de la digue est finalement établi. 


\section{XVIèmes Journées Nationales Génie Côtier - Génie Civil \\ Le Havre 2020}

\section{Le projet de digue de Xiapu}

2.1 Deux corps de digue : avec ou sans apport de matériaux traditionnels ?

Le projet de digue fait partie de la construction d'un second port protégé de l'île de Zhujiang dans la ville de Shajiang (district de Xiapu, province du Fujian). La structure hydraulique principale est une digue d'environ $2 \mathrm{~km}$, d'une hauteur de $5.5 \mathrm{~m}$ et largeur maximale de $7.9 \mathrm{~m}$. Dans le projet à l'origine, le corps de digue était à base de matériaux naturels classiques (figure 1a). Il a été modifié par la suite pour minimiser l'apport de matériaux et valoriser les sédiments de proximité en tant que matériaux (figure 1b).

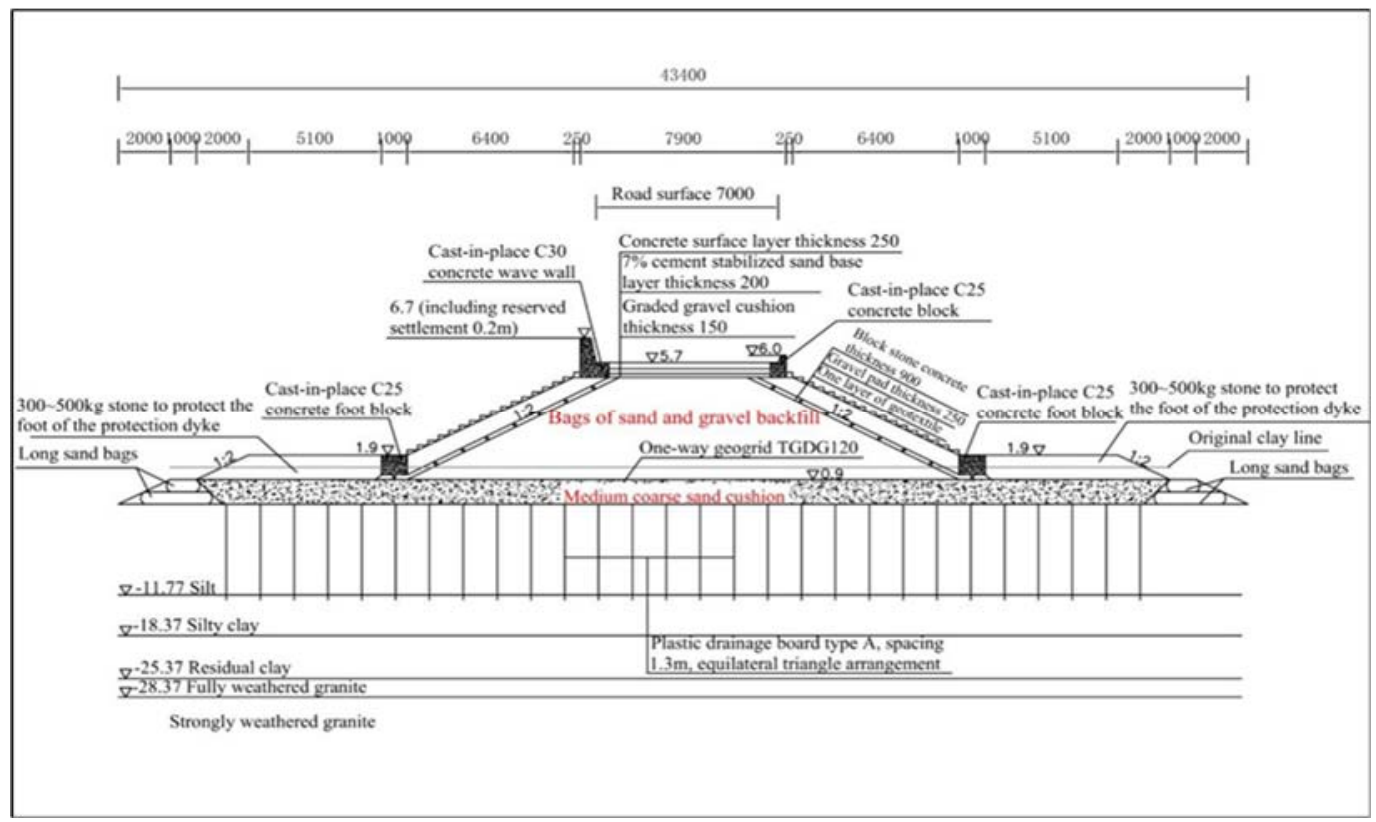

a - Noyau avec matériaux naturels, concept d’origine.

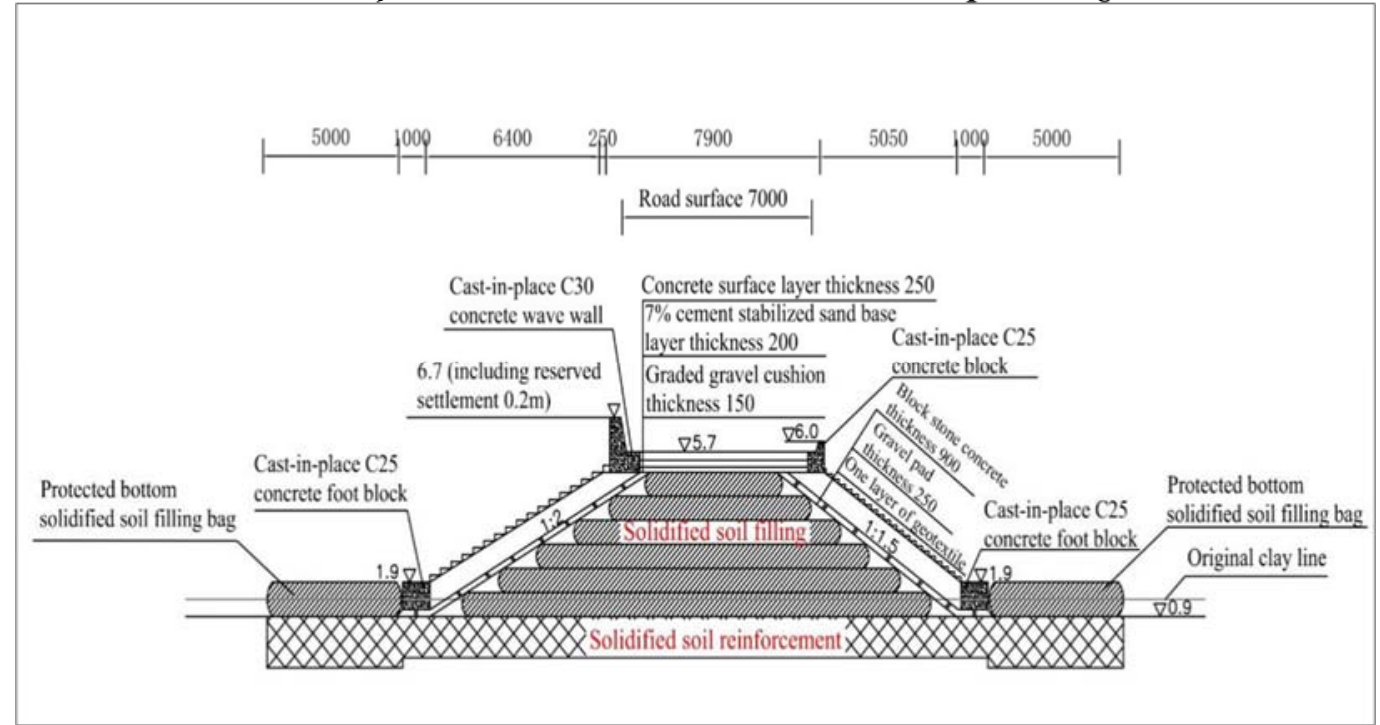

b - Noyau en sacs en géotextile remplis de sédiments solidifiés, concept modifié. Figure 1. Coupes de la digue de Xiapu. 


\section{Thème 6 - Gestion durable des zones littorales et estuariennes}

\subsection{Géologie et caractéristiques géotechniques du site}

Une série de 6 forages a permis de reconnaitre les couches principales de sol le long de la digue projetée. En surface, une couche de vase limoneuse épaisse d'environ $12 \mathrm{~m}$ surplombe une couche d'argile limoneuse et d'argile se situant entre $-12 \mathrm{~m}$ et $-25 \mathrm{~m}$. Ces couches se positionnent sur un granite plus ou moins altéré. Les propriétés physiques et mécaniques sont pour une part rassemblées dans le tableau 1.

Tableau 1. Données géotechniques des couches de sol le long du projet de digue.

\begin{tabular}{|c|c|c|c|c|c|c|c|c|c|c|c|}
\hline $\begin{array}{c}\text { Couche } \\
\text { de sol }\end{array}$ & $\begin{array}{c}\text { Profondeur } \\
(\mathrm{m})\end{array}$ & $\begin{array}{c}w \\
(\%)\end{array}$ & $\begin{array}{c}\rho \\
\left(\mathrm{g} / \mathrm{cm}^{3}\right)\end{array}$ & $e_{0}$ & $\begin{array}{c}W_{L} \\
(\%)\end{array}$ & $\begin{array}{c}W_{P} \\
(\%)\end{array}$ & $I L$ & $I P$ & $\begin{array}{c}E_{M} \\
(\mathrm{MPa})\end{array}$ & $\begin{array}{c}c \\
(\mathrm{kPa})\end{array}$ & $\begin{array}{c}\varphi \\
\left({ }^{\circ}\right)\end{array}$ \\
\hline $\begin{array}{c}\text { Silt } \\
\text { de surface }\end{array}$ & $0-12$ & 59.23 & 1.60 & 1.64 & 53.39 & 31.06 & 1.25 & 22.33 & 1.80 & 10.53 & 7.20 \\
\hline $\begin{array}{c}\text { Argile } \\
\text { limoneuse }\end{array}$ & $12-20$ & 21.69 & 1.95 & 0.68 & 31.09 & 18.14 & 0.27 & 12.95 & 5.61 & 27.87 & 21.25 \\
\hline $\begin{array}{c}\text { Argile } \\
\text { résiduelle }\end{array}$ & $20-25$ & 24.43 & 1.87 & 0.78 & 32.17 & 20.77 & 0.32 & 11.40 & 5.18 & 18.68 & 18.81 \\
\hline
\end{tabular}

Note : $W$ teneur en eau, $\rho$ masse volumique humide, $e_{0}$ indices des vides naturel, $W_{\mathrm{L}}$ limite de liquidité, WP limite de plasticité, $I L$ indice de liquidité, IP indice de plasticité, $E_{M}$ module de compressibilité, c cohésion, $\varphi$ angle de frottement interne.

\subsection{Caractéristiques exigées pour le sédiment solidifié dans le projet}

La modification du projet de digue de Xiapu a imposé un changement de concept (figure 1b) et le sédiment de proximité a été traité pour qu'il soit réutilisé dans la construction de 200m de digue comme matériau en substitution aux matériaux naturels. Il a servi de matériau d'assise ou fondation et il a constitué le matériau du corps de digue par remplissage de sacs de géotextile. Les performances du sédiment traité à l'état frais (pompage et transport) et durci à 28 jours sont données dans le tableau 2.

Tableau 2. Exigences de performance pour le sédiment solidifié pour le corps de digue.

\begin{tabular}{|c|c|c|c|}
\hline \multirow{4}{*}{$\begin{array}{l}\text { Performance } \\
\text { du sédiment solidifié } \\
\text { à l'état frais }\end{array}$} & \multirow{2}{*}{ Affaissement A (mm) } & Initial & \multirow{2}{*}{$110 \mathrm{~mm} \leq A \leq 150 \mathrm{~mm}$} \\
\hline & & $1 \mathrm{~h}$ & \\
\hline & \multirow{2}{*}{ Temps de prise (min) } & Consistance initiale & $\geq 180$ \\
\hline & & Consistance finale & $\leq 600$ \\
\hline \multirow{4}{*}{$\begin{array}{l}\text { Propriétés } \\
\text { du sédiment solidifié }\end{array}$} & $U C S_{28}$ jours $(\mathrm{kPa})$ & - & $\geq 120$ \\
\hline & \multirow{2}{*}{$\begin{array}{l}\text { Paramètres de } \\
\text { cisaillement à } 28 \text { jours }\end{array}$} & $c(\mathrm{kPa})$ & $\geq 20$ \\
\hline & & $\varphi\left(^{\circ}\right)$ & $\geq 15$ \\
\hline & $\begin{array}{l}\text { Coefficient de perméabilité } \\
\text { verticale } k_{v}(\mathrm{~cm} / \mathrm{s})\end{array}$ & $k_{v}$ & $<1.0 \times 10^{-5}$ \\
\hline
\end{tabular}

Note : UCS résistance en compression simple.

Le sédiment traité est un sédiment prélevé sur le site du projet dans la couche de surface. C'est une argile plutôt limoneuse de consistance plastique à fluide et relativement homogène.

Le liant ou agent de durcissement, référencé H01 est fourni par la société Jiangsu Kun Ze Technologie Co., Ltd. Il s'agit d'un mélange de laitier, de clinker, de cendres volantes et de gypse. 


\section{XVIèmes Journées Nationales Génie Côtier - Génie Civil \\ Le Havre 2020}

\section{Etude de la fluidité du sédiment traité à l'état frais}

\subsection{Paramètres retenus dans l'étude}

Le mélange sédiment-eau-liant doit rester suffisamment fluide pour assurer son transport et mise en place avant durcissement. La fluidité du mélange dépend d'une part de la nature du sédiment, de sa teneur en eau et du dosage en liant mais de facteurs liés au chantier (méthode i.e. avec ou sans agitation et durée de stockage, température). Des essais croisés effectués sur le sédiment de surface mais de nature différenciée (argile, argile limoneuse, limon) ont montré que l'importance des facteurs influençant l'affaissement initial, sont dans l'ordre la teneur en eau du mélange (w variant de 100 à 140\%), la nature du sédiment (3 types) et le dosage en liant ( 80 à $\left.120 \mathrm{~kg} / \mathrm{m}^{3}\right)$, (WANG, 2019).

\subsection{Effets de la teneur en eau du mélange des sédiments sur la fluidité}

La fluidité ou consistance du mélange sédiment-eau-liant a été évaluée à partir de la mesure de l'affaissement au cône (facilité d'utilisation en chantier). Il a été montré que la teneur en eau fait augmenter l'affaissement mais différemment selon le temps écoulé depuis le temps initial to. Un exemple d'évolution est donné sur la figure 2, sur laquelle on observe deux comportements avant et après $150 \%$ de teneur en eau pour un mélange dosé à $100 \mathrm{~kg} / \mathrm{m}^{3}$. Selon les exigences du projet, un affaissement A doit être compris entre $110 \mathrm{~mm}$ et $150 \mathrm{~mm}$ (tableau 2), ce qui entraîne que la teneur en eau du mélange à l'état frais doit être comprise entre 120 et $150 \%$.

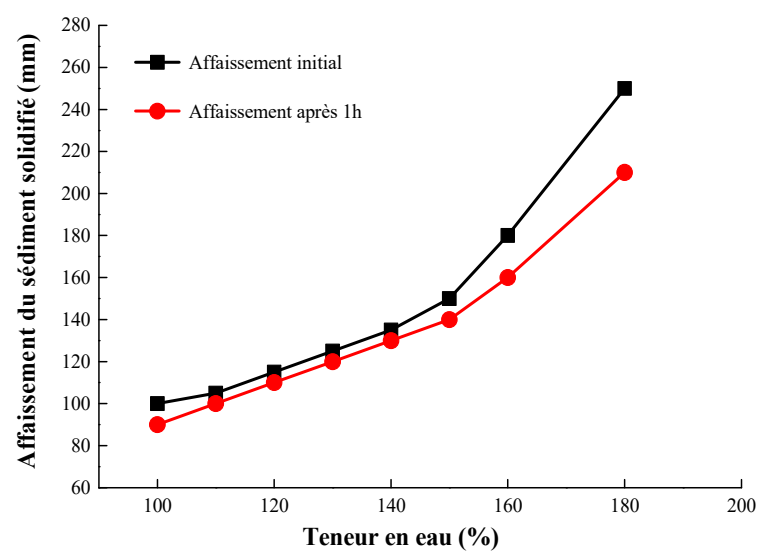

Figure 2. Effet de la teneur en eau sur l'affaissement du sédiment traité.

\subsection{Influence de la méthode et durée de stockage et de la température}

L'affaissement i.e. la consistance du mélange dépend du mode de stockage, de sa durée et de la température au moment de la préparation, du transport et de la mise en place. On peut observer ces influences pour le sédiment traité à $150 \%$ de teneur en eau et dosé à $100 \mathrm{~kg} / \mathrm{m}^{3}$ en liant sur les figures 3 pour 2 modes de stockage : avec ou sans agitation à $20^{\circ} \mathrm{C}$ (figure $3 \mathrm{a}$ ) ; pour des températures variant de 20 à $30^{\circ} \mathrm{C}$ avec agitation continue 


\section{Thème 6 - Gestion durable des zones littorales et estuariennes}

(figure $3 \mathrm{~b}$ ) et des teneurs en eau allant de 120 à $150 \%$ sous agitation continue (figure $3 \mathrm{c}$ ). On remarque que la perte d'affaissement dans le temps est d'autant plus marquée sans l'agitation du mélange (figure 3a). Ceci est d'importance dans la préparation du mélange et pendant le processus de pompage en chantier. Une autre observation concerne la perte d'affaissement pour une température supérieure à $30^{\circ} \mathrm{C}$ (figure $3 \mathrm{~b}$ ). A une température de $20^{\circ} \mathrm{C}$, sous agitation continue, au fur et à mesure du temps écoulé depuis to, l'affaissement est réduit quelle que soit la teneur en eau mais au-delà d'une heure, cette réduction est notable (figure 3c).
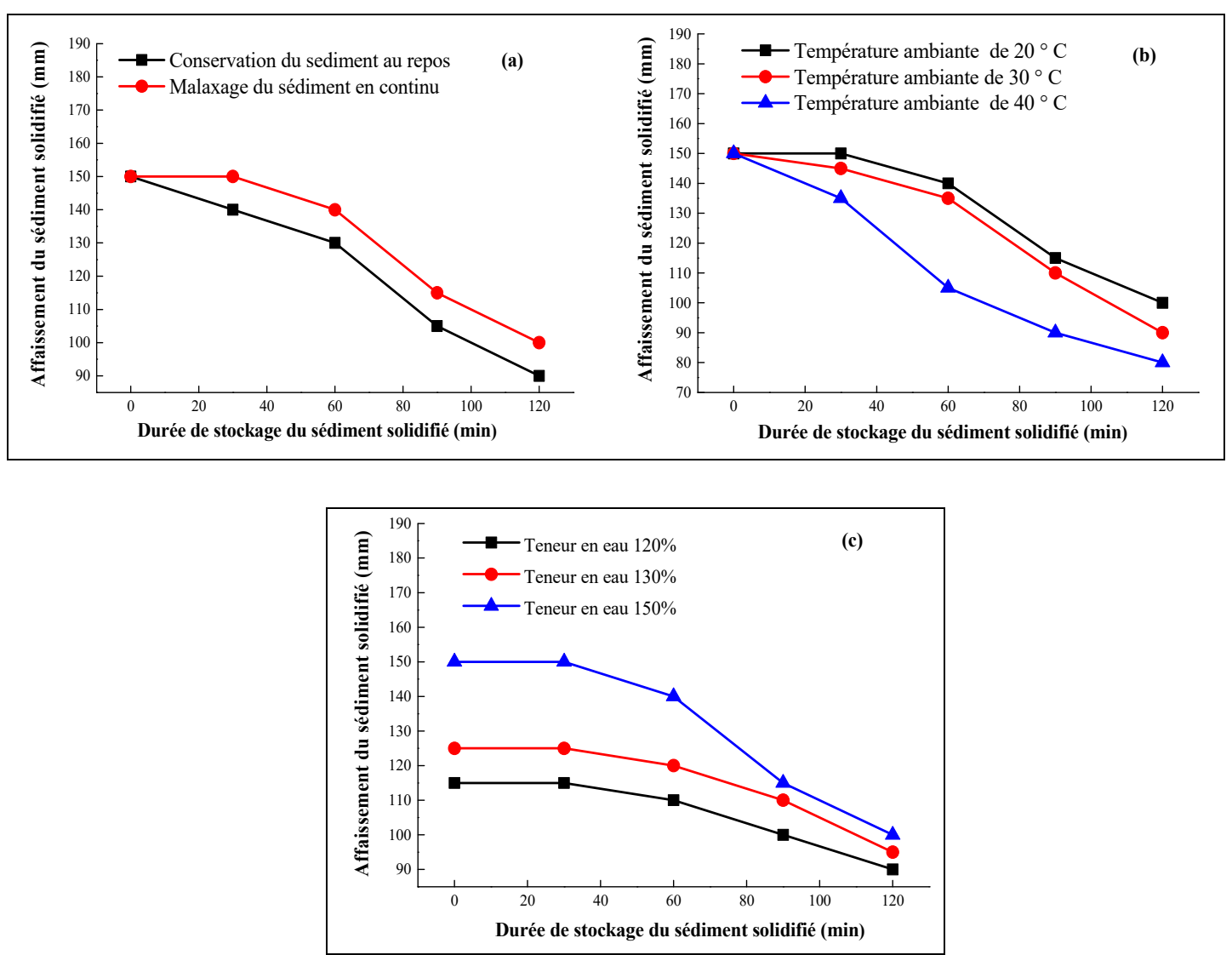

Figure 3. Effets du mode et de la durée de stockage, de la température sur l'affaissement du sédiment traité.

Du point de vue pratique, ces observations ont permis de déterminer que, pour que la fluidité du sédiment soit conforme aux exigences de la construction dans le cas de la digue de Xiapu, la température en chantier ne devrait pas dépasser $30^{\circ} \mathrm{C}$ et que le sédiment traité devrait être préparé et stocké avec une agitation continue. La teneur en eau du mélange à respecter serait d'environ $120 \%$ à $150 \%$ et le temps de mise en place du sédiment traité se situe à 1,5 heure. 


\section{XVIèmes Journées Nationales Génie Côtier - Génie Civil \\ Le Havre 2020}

\section{Etude du sédiment traité à l'état durci à 28 jours}

\subsection{Paramètres retenus dans l'étude de laboratoire}

L'état durci du mélange sédiment-eau-liant a été étudié en laboratoire et les essais à 28 jours ont porté sur les mesures de résistance mécanique comme la résistance en compression simple (UCS), la cohésion c, l'angle de frottement interne $\varphi$ et la mesure de la perméabilité verticale $\mathrm{k}$. Ces paramètres ont été évalués en fonction de la teneur en eau et du dosage en liant comme indiqué dans le tableau 3. Il s'avère que ces résultats pour un dosage de $100 \mathrm{~kg} / \mathrm{m}^{3}$ et une teneur en eau entre 130 et $140 \%$ satisfont les exigences de conception demandées.

Tableau 3. Caratéristiques hydromécaniques du sédiment solidifié en laboratoire.

\begin{tabular}{|c|c|c|c|c|c|}
\hline \multirow{2}{*}{$W(\%)$} & \multirow{2}{*}{$\begin{array}{c}\text { Dosage en liant } \\
\left(\mathrm{kg} / \mathrm{m}^{3}\right)\end{array}$} & \multicolumn{4}{|c|}{ Propriétés du sédiment solidifié à 28 jours } \\
\hline & & UCS (kPa) & $c(k P a)$ & $\varphi\left({ }^{\circ}\right)$ & $k_{v}(\mathrm{~cm} / \mathrm{s})$ \\
\hline \multirow{3}{*}{120} & 80 & 179 & 29.4 & 23.2 & $5.7 \times 10^{-6}$ \\
\hline & 100 & 206 & 31.6 & 25.2 & $5.8 \times 10^{-6}$ \\
\hline & 120 & 312 & 40.3 & 27.1 & $6.0 \times 10^{-6}$ \\
\hline \multirow{3}{*}{130} & 80 & 139 & 25.3 & 22.5 & $6.1 \times 10^{-6}$ \\
\hline & 100 & 193 & 29.2 & 25.1 & $6.4 \times 10^{-6}$ \\
\hline & 120 & 242 & 32.4 & 26.2 & $6.2 \times 10^{-6}$ \\
\hline \multirow{3}{*}{140} & 80 & 123 & 24.0 & 22.4 & $6.8 \times 10^{-6}$ \\
\hline & 100 & 169 & 28.7 & 24.7 & $7.2 \times 10^{-6}$ \\
\hline & 120 & 201 & 30.2 & 25.6 & $7.9 \times 10^{-6}$ \\
\hline \multirow{3}{*}{150} & 80 & 109 & 18.2 & 19.1 & $8.8 \times 10^{-6}$ \\
\hline & 100 & 131 & 19.1 & 20.2 & $9.2 \times 10^{-6}$ \\
\hline & 120 & 162 & 21.2 & 20.5 & $2.1 \times 10^{-5}$ \\
\hline
\end{tabular}

\subsection{Paramètres mesurés sur site}

A l'achèvement de la digue de Xiapu, à la date standard de 28 jours, les essais d'acceptation géotechnique pour le sédiment solidifié du corps de la digue ont été effectués par un bureau de contrôle extérieur. Pour ces essais de contrôle, le corps de la digue a été divisé en 6 couches de bas (couche 1) en haut (couche 6) pour construire le noyau, voir figure $2 \mathrm{~b}$. Le sédiment solidifié est prélevé dans chaque couche (voir photo du tableau 3 ) et soumis à aux essais d'acceptation du site dont les résultats sont récapitulés dans le tableau 3. Pour l'ensemble des essais de contrôle de tous les échantillons prélevés sur l'ouvrage réalisé, il a été observé que la cohésion, l'angle de frottement, le coefficient de perméabilité verticale et la résistance à la compression non confinée dans chaque couche de sédiment solidifié satisfont aux exigences de conception confirmant la qualité de la construction du noyau en sédiments solidifiés. 


\section{Thème 6 - Gestion durable des zones littorales et estuariennes}

Tableau 4. Caratéristiques hydromécaniques du sédiment solififié sur site.

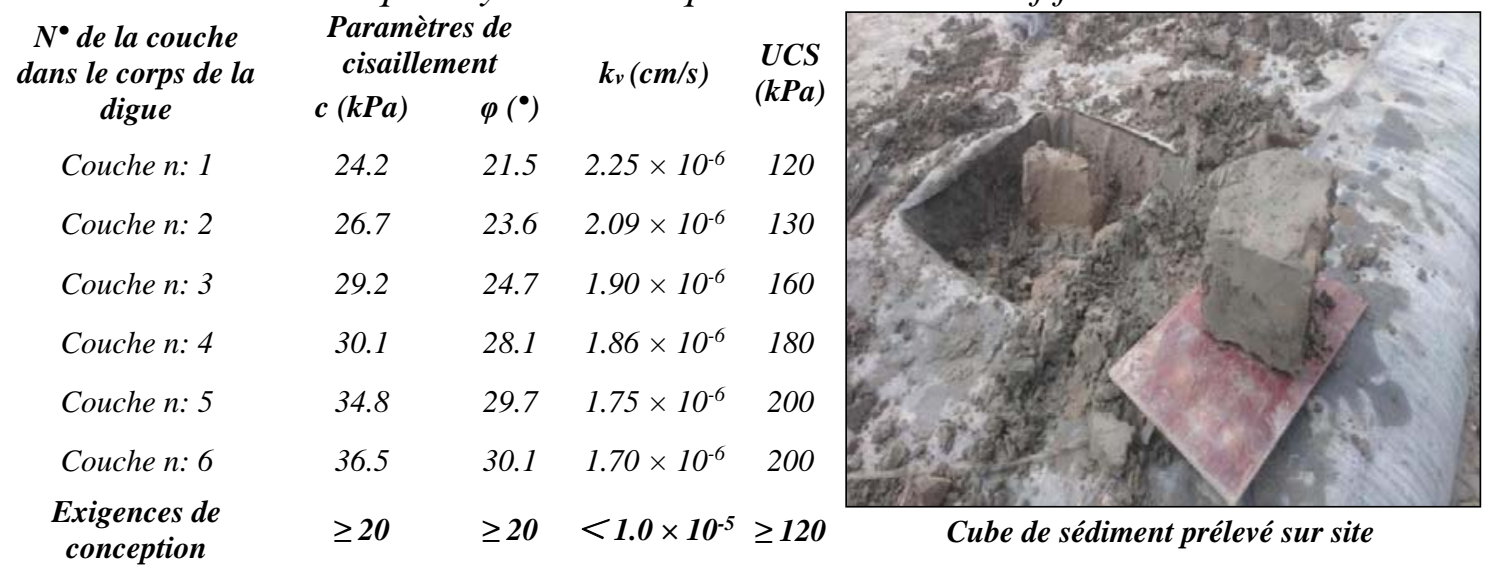

\section{Conclusions}

Le sédiment i.e. argile limoneuse a été mis en œuvre avec une teneur élevée (130 à 140\%) mélangé avec un liant spécial dosé à $100 \mathrm{~kg} / \mathrm{m}^{3}$. Ce mélange a été pompé et a rempli les sacs en géotextiles i.e. géotubes ${ }^{\circledR}$ prévus pour le corps de digue en 6 couches (voir figure 4). Une technologie appropriée a été développée pour réaliser le pompage et un remplissage $(\geq 70 \%)$. Les exigences de conception ont pu être satisfaites à la suite d'essais spécifiques menés en laboratoire confrontés à des essais de contrôle sur site.
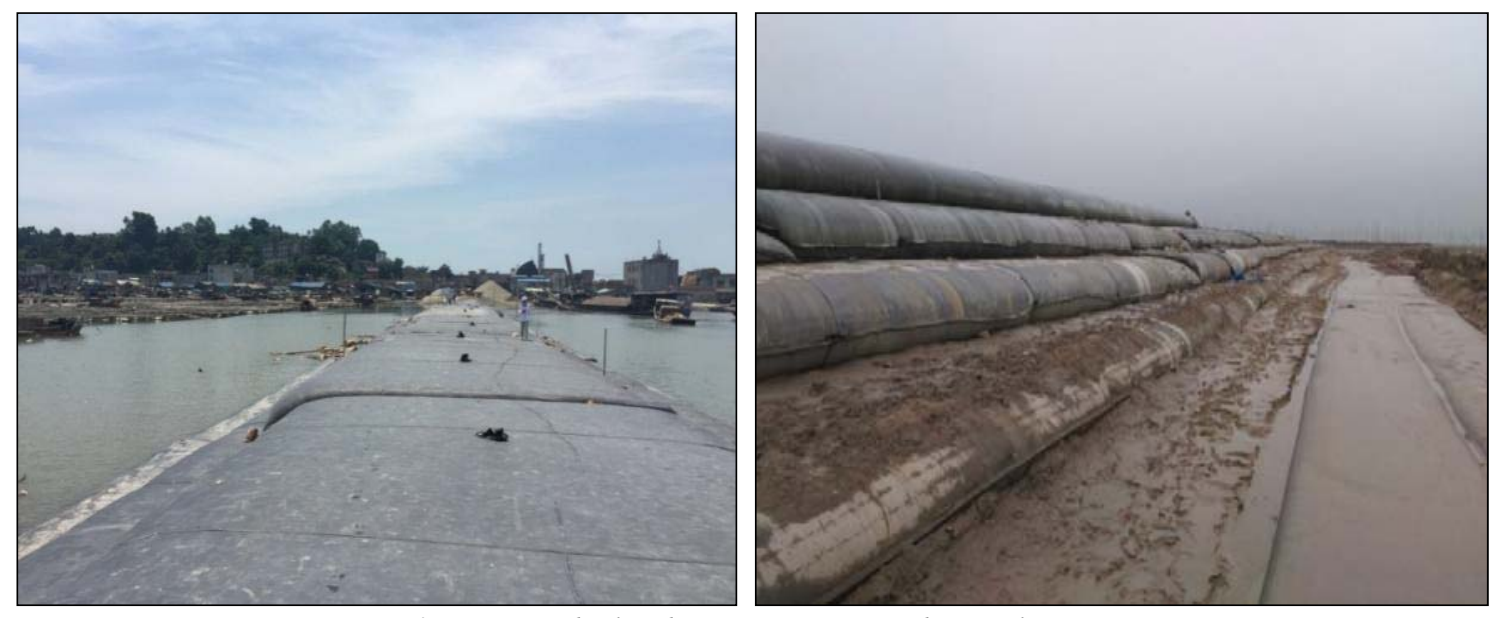

Figure 4. Corps de la digue en cours de réalisation.

\section{Références}

PU S.Y., ZHU Z.D., WANG H.R., SONG W.L., WEI R.J. (2019). Mechanical characteristics and water stability of silt solidified by incorporating lime, lime and cement mixture, and SEU-2 binder, Construction and Building Materials, Vol. 214, pp 111-120. https://doi.org/10.1016/j.conbuildmat.2019.04.103

WANG X. (2019). Méthodologies de valorisation de sédiments mises en œuvre dans des ouvrages géotechniques en Chine. Thèse de doctorat, U. de Caen Normandie, 202 p. 